\title{
A Comparison of Single Reference Station, Correction-Based Multiple Reference Station, and Tightly Coupled Methods using Stochastic Ionospheric Modelling
}

\author{
P. Alves and G. Lachapelle \\ Position, Location And Navigation Group, Department of Geomatics Engineering, University of Calgary, Canada \\ Tel: (1-403) 210 9796, Fax: (1-403) 2841980, Email: lachapelle@geomatics.ucalgary.ca
}

Received: 6 December 2004 / Accepted: 12 July 2005

\begin{abstract}
The multiple reference station approach to carrier phase-based positioning uses a network of GPS reference stations to model the correlated errors in a geographic region. This paper compares two methods for multiple reference station positioning under a low and a high level of ionosphere. The first method tested is the conventional method for multiple reference station positioning, which is usually a three-step process, namely (1) estimation of the carrier phase ambiguities in the network, (2) prediction of the measured network errors at the location of the rover, and (3) application of the corrections in a practical format. The second method is called the tightly coupled or in-receiver approach, which uses the data from the rover and integrates it with the network solution to better model the effect of the ionosphere. In this approach there are no explicit corrections. These two methods are compared with the single reference station approach for data from two days collected from the Southern Alberta Network in Canada, a medium scale network with inter-stations distance of 34 to $59 \mathrm{~km}$.
\end{abstract}

Key words: Multiple reference station GPS RTK, VRS, single reference station GPS, least squares collocation

\section{Introduction}

Network RTK (Real Time Kinematic) implementation consists of three main steps (Lachapelle and Alves, 2002). In the first step, the errors at the reference stations are estimated using carrier phase observations. The second step interpolates these errors to the rover receiver location and finally the corrections are transmitted to the rover in the third step. This process is usually carried out through the generation of virtual reference station (VRS) data that the rover can accept in a single reference station data format. In this way, standard single reference station RTK software can be used at the receiver.

Real-time kinematic network positioning is limited by many factors, one of which is the communication network used between the network control centre and the rovers. Due to bandwidth limitations with multiple rovers and an attempt to allow for user privacy, network RTK positioning methods have attempted to operate a broadcast-only system (one-way communication), whereby the network corrections are broadcast to all rovers and there is no information communicated from the rover back to the network.

If alternate communication methods are used then not only can the network stations assist the rover but the rover can also assist the network with additional information. In this case the rover actually becomes part of the network and the reduced inter-receiver distances and additional ambiguity constraints provided by the rover improve the overall ambiguity resolution process very significantly using the now established receiver multiplicity; concept initially proposed by Lachapelle et al. (1993) and further tested by Luo and Lachapelle (2003). This enhanced procedure is also ideal for postmission applications such as, verification of hydrographic surveys, airborne surveys, and land surveying.

Network RTK systems use reference stations to precisely measure the correlated errors affecting the region. These errors can only be measured when all other parameters are precisely determined, namely the station position and carrier phase ambiguities. With this in mind, the better a station's position and ambiguities are known, the more accurately one can separate measurement errors and 
systematic biases. Reference stations are an obvious choice because their positions are known, but any receiver can be used to estimate measurement errors. For example, a static or kinematic rover can be treated as a reference station. In terms of error modelling, multiple rovers in an area can each give an indication of the local environmental error conditions, e.g. the state of the ionosphere that is a major error source. Combining all of this information into a coherent model allows for new network rovers, with less defined position and velocity estimates, to benefit from decreased measurement errors.

The assistance of the rover to the network can be seen in the baseline configurations for the network. Ambiguity resolution performance is a function of the inter-antenna distance separation because the correlated errors increase in magnitude as the inter-antenna distance increases. In a broadcast-only Network RTK system, baselines are formed between the various reference stations. Rovers within the network will, by definition, be between two or more reference stations. Therefore connecting baselines to the rovers as well as the reference stations will shorten the overall network inter-antenna separations within the network, thus giving a higher likelihood of resolving the carrier phase ambiguities.

Instead of applying a simple weighted average (prediction) approach, the rover's data and estimated states are added to the network filter. The network filter is used solely to estimate and resolve the network ambiguities in the real-time approach. The addition of the rover's information into the network filter maintains all the information used in the correction-based approach and adds the rover's measurements. The difference is that the network not only assists the rover but the rover also assists the network.

In the loosely coupled (correction-based) approach, network ambiguities (and other nuisance parameters, such as the ionosphere) are estimated using Bayes filtering. The ambiguity estimates are then searched, and if validated, resolved. The resulting ambiguities and measurement residuals are then used to predict the errors at the rover locations.

The integrated approach does not require the error prediction phase of the loosely coupled approach because the error estimates are reduced from the rover's estimates when the rover's position is estimated. This is accomplished by the signal covariance function, which is used to evaluate the contribution of each of the reference station's observations on the rover. The covariance function is a statistical measure of the correlated errors, namely orbital, tropospheric, and ionospheric errors, between measurements. If two measurements are highly correlated, then when they are differenced, the variance of the resulting differenced observation is reduced. Consider the case where the rover is connected to every baseline in the network. The reference station observations that are highly correlated with the rover are assigned a low variance and as a result, are given more weight in the adjustment than an observation whose errors are different than those of the rover. This method of weighting produces an error model using all of the surrounding reference station data.

Pugliano et al. (2003, 2004), Alves (2004), and Alves et al. (2004), show significant improvements when using the tightly coupled approach on a variety of rover and network configurations.

\section{Tightly Coupled Implementation}

The design matrix of the tightly coupled (in-receiver) approach has the form

$$
A=\left[\begin{array}{ccccccccc}
\frac{\partial \Delta \nabla \Phi_{1}}{\partial x} & \frac{\partial \Delta \nabla \Phi_{1}}{\partial y} & \frac{\partial \Delta \nabla \Phi_{1}}{\partial z} & \lambda & 0 & & 0 & 0 & \\
\frac{\partial \Delta \nabla \Phi_{2}}{\partial x} & \frac{\partial \Delta \nabla \Phi_{2}}{\partial y} & \frac{\partial \Delta \nabla \Phi_{2}}{\partial z} & 0 & \lambda & & 0 & 0 & \\
0 & 0 & 0 & 0 & 0 & & \lambda & 0 & \\
0 & 0 & 0 & 0 & 0 & & 0 & \lambda & \\
0 & & & & & & & &
\end{array}\right]
$$

where the first n rows correspond to the double difference observations between the rover and one of the reference stations and the second set of $m$ rows correspond to double difference observations between the fixed reference stations with known coordinates. $\mathrm{n}$ is the number of double difference observations between the rover and the reference station(s) and $m$ is the number of double difference observations between reference stations. The first three columns correspond to the rover's position estimates and the following $n+m$ columns correspond to the ambiguities of all the double difference observations. $\Phi$ represents the carrier phase measurements in unit of length (m) and $\lambda$ is the measurement wavelength in unit of length $(\mathrm{m})$. The partial derivatives of the coordinates with respect to the reference stations are zero because the reference station coordinates are known and held fixed.

The design matrix can be extended to accept any number of reference stations and rovers. The processing shown includes the code and carrier phase observations processed in a single Bayes filter. This model can be expanded to incorporate any observation (system) model (estimating atmospheric errors and/or the rover's velocities, for example). The selection of the double difference observables is based on the shortest interreceiver separations, with the conditions of linear independence and connectivity being preserved. Thus a rover may be connected to one or several reference stations, depending on the reference station-rover receiver 
configuration. Short distances are selected to minimize the magnitude of the differential errors. As an example, in the case of four reference stations and one rover, the double differences over the shortest four linearly independent receiver separations would be used. The rover may be involved in one to four sets of double differences.

In order to maintain the information from the correctionbased approach, mathematical and stochastic information must be preserved in the integrated approach. The mathematical correlation is due to inter-receiver separations that share a common reference station (or rover) that uses the same observations in the double difference measurements. This is represented in the filter by the double difference measurement matrix, B. This matrix is not block diagonal because the observations from one station may be used in multiple baselines. The measurement matrix for the scenario where there are four stations and each station is used in a maximum of two baselines is

$$
B=\left[\begin{array}{cccc}
B_{s d} & -B_{s d} & 0 & 0 \\
0 & B_{s d} & -B_{s d} & 0 \\
0 & 0 & B_{s d} & -B_{s d}
\end{array}\right]
$$

where $B_{s d}$ is the single difference measurement matrix for each of the stations, assuming that each station has the same satellites in view. This correlation is often neglected in multiple baseline processing.

\section{Correction-Based Method}

The tightly coupled approach described above is compared herein to the typical correction-based multiple reference station approach. The correction-based model uses the network ambiguities to measure the residual errors. These residuals are interpolated to the location of the rover station using an interpolating function. In this case least-squares collocation is used to interpolate (or predict) the reference station residuals to the location of the rover. The corrections are calculated as

$$
\begin{aligned}
& \hat{\delta} \hat{\mathbf{l}}=C_{l} B^{T}\left(B C_{l} B^{T}\right)^{-1}(B \Phi-\Delta \nabla \mathbf{N}) \\
& \delta \hat{\mathbf{l}}_{r}=C_{l_{r}, l} B^{T}\left(B C_{l} B^{T}\right)^{-1}(B \Phi-\Delta \nabla \mathbf{N})
\end{aligned}
$$

where $\delta \hat{\mathbf{l}}$ is the correction vector, $C$ is a covariance matrix, $B$ is the observation double difference matrix, $\Phi$ is a vector of the carrier phase ambiguities minus range, and $\Delta \nabla \mathbf{N}$ is a vector containing the double difference carrier phase ambiguities in unit of length. The subscript " $r$ " refers to the measurements of the reference station used in the single difference processing. This method was introduced by Raquet (1998).

\section{Covariance Function}

Stochastic correlation is defined by the signal covariance function. The covariance function states the likelihood of two values being the same based on a physical process. For example, it is known that the ionosphere is a spatially correlated error, therefore two stations close to each other are likely to have a similar ionospheric error. Stochastic correlation is represented in the Bayes filter in the variance-covariance matrix of the observations. This is a fully populated matrix because each of the measurement pairs should be somewhat correlated. The following covariance function form is used

$$
\sigma_{a, b}=e^{-\frac{d}{\beta_{d}}} e^{-\frac{\alpha}{\beta_{\alpha}}} \sigma_{T}^{2}+e^{-\frac{d_{I}}{\beta_{d_{I}}}} \sigma_{I}^{2}
$$

where the covariance between observations $a$ and $b\left(\sigma_{a, b}\right)$ is a function of the distance, $d$, between the two reference stations, the great circle angle, $\alpha$, between the measurements and the ionosphere pierce point distance, $d_{I} . \quad \sigma_{T}^{2}$ and $\sigma_{T}^{2}$ are the variances of the troposphere and ionosphere components respectively. The parameters of the covariance function $\left(\beta_{d}, \beta_{\alpha}, \sigma_{T}^{2}, \beta_{d_{I}}\right.$, and $\left.\sigma_{I}^{2}\right)$ are estimated in real-time so that they can adaptively respond to changing atmospheric conditions, as proposed by Alves (2004).

\section{Ionosphere Modelling}

Stochastic ionospheric modelling is used to reduce the effect of the ionosphere on the estimated position and ambiguities. This model estimates the dual frequency slant ionospheric delays using the ionosphere-free model (Odijk, 1999). In addition to the rover's position, velocity, and ambiguity states, an ionospheric parameter for each dual frequency satellite pair is estimated. The corresponding rows and columns are added to the design matrix in Equation 1.

The following description is in terms of the tightly coupled approach, however the single reference station approach uses the same methodology except that only one reference station is used. The design matrix is partitioned into sub-matrices as

$$
A=\left[\begin{array}{ll}
A_{(1,1)} & A_{(1,2)} \\
A_{(2,1)} & A_{(2,2)}
\end{array}\right]
$$

where the first row of two matrices refers to the measurements of baselines that include the rover as one of the stations. The second row refers to measurements of baselines with only network stations. As there are no common estimated parameters between the network and rover, 


$$
\begin{aligned}
& A=A_{(1,2)}=A_{(2,1)}=0 \\
& A_{(1,1)}=\left[\begin{array}{cccc}
A_{\text {pos }} & \lambda_{L 1} I & 0 & I \\
A_{\text {pos }} & 0 & \lambda_{L 2} I & \frac{f_{L 1}^{2}}{f_{L 2}^{2}} I
\end{array}\right]
\end{aligned}
$$

where the two rows of the above sub matrix represent the two measurement types used, namely L1 and L2 phase measurements. $\quad A_{\text {pos }}$ is a 3-column sub-matrix that includes the partial derivatives of the double difference measurements with respect to the three position components of the rover. If the velocity is estimated, then $A_{\text {pos }}$ contains six columns where the last three columns are zero. $f$ is the measurement frequency in Hertz and $I$ is the identity matrix.

The sub matrix for the network observations has the same form as the sub matrix for the rover observations. The difference is that the first row is removed as shown.

$$
A_{(2,2)}=\left[\begin{array}{ccc}
\lambda_{L 1} I & 0 & I \\
0 & \lambda_{L 2} I & \frac{f_{L 1}^{2}}{f_{L 2}^{2}} I
\end{array}\right]
$$

The first column is removed because no position states are measured by the network observations. Each row of this sub matrix represents the same observation types as the rover's observations.

\section{Test Methodology}

The performance evaluation of the three methods, correction-based multiple reference station approach, tightly coupled approach, and the single reference station approach is broken down into two parts, namely convergence and steady state. To test the convergence performance of each of the methods, the processing filters are reset each hour. The rover position is initialized with an error of $1 \mathrm{~m}$ when the filters are reset. This process gives 24 convergence trials over a period of 24 hours. Each epoch during convergence is averaged across the trials. The root mean squared (RMS) position error after convergence is presented as an indication of the steady state position accuracy. A data rate of 30 seconds is used in all of the tests.

The use of stochastic ionospheric modelling is also evaluated in this study. To simulate realistic real-time operational performance, the network correction computation always implements stochastic ionospheric modelling. Although the rovers used in these tests are in static mode, they have been processed in kinematic mode, i.e. no batch solution. The positions and velocities are estimated as a first order Gauss-Markov process.
MultiRef ${ }^{\mathrm{TM}}$ and MultiRefPM ${ }^{\mathrm{TM}}$ were used for processing (e.g., Alves et al 2004). These software packages were developed at the University of Calgary. The MultiRef ${ }^{\mathrm{TM}}$ software was used to calculate the network corrections for the correction-based approach. All of the rover positions are calculated using MultiRefPM ${ }^{\mathrm{TM}}$. To ensure that the same processing methods are used for all of the compared approaches, the tightly coupled algorithm is used for processing in all cases, however only one baseline is processed in the single and correction-based scenarios.

MultiRef $^{\mathrm{TM}}$ and MultiRefPM${ }^{\mathrm{TM}}$ estimate both L1 and L2 ambiguities with the option of implementing stochastic ionosphere modelling. The best available ambiguities (be it fixed for float) are used for the final positioning solution and correction computation.

\section{Southern Alberta Network}

The Southern Alberta Network (SAN) was used to conduct the performance analysis. This network of dualfrequency NovAtel Modulated Precision Clock (MPC) receivers is operated and maintained by the PLAN Group of the University of Calgary. The network of 14 reference stations covers approximately $150 \mathrm{~km}$ north-south and $200 \mathrm{~km}$ east-west around Calgary. It is used in a variety of GPS research projects at the University of Calgary including Network RTK and GPS meteorology.

The sub-network shown in Figure 1 was used herein to evaluate the improvement due to the in-receiver multiple reference station approach whereby UOFC is considered the roving receiver. The baselines used for the inreceiver approach are the shortest set of independent baselines. The baseline lengths of the network range from 24 to $49 \mathrm{~km}$. The closest reference station is used to evaluate the performance of the single reference station approach. This creates a single reference station interantenna distance of approximately $24 \mathrm{~km}$. 




Fig. 1 Network configuration for the in-receiver multiple reference station approach with UOFC as the rover

The network configuration shown in Figure 2 is used for the correction-based multiple reference station approach. None of the network baselines connect to the rover in this approach, which makes the baseline lengths slightly longer. The baseline lengths range from 34 to $59 \mathrm{~km}$ for the correction-based approach.



Fig. 2 Network configuration for the correction-based multiple reference station approach with UOFC as the rover

Two days of data were used for the performance evaluation, namely May 24 and April 6, 2004. These were selected because they represent a relatively low and high level of ionospheric error respectively. Figure 3 shows the estimated double difference slant ionospheric effect for a $60 \mathrm{~km}$ baseline for the two days. The ionosphere is more variable and higher in magnitude for April 6 (bottom) then for May 24 (top). On April 6, the double-difference ionospheric effect reaches about 5 ppm.
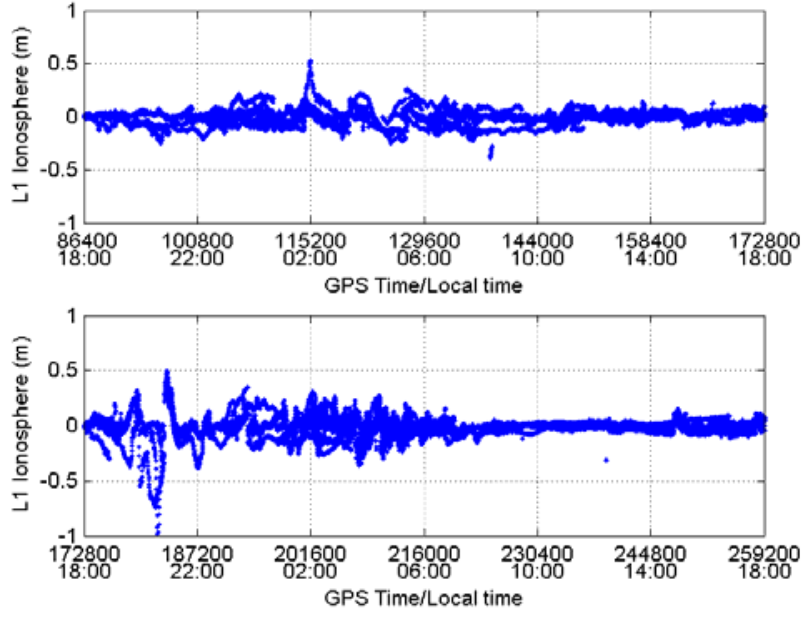

Fig. 3 Estimated double difference slant ionosphere for May 24 (top) and April 6 (bottom) for a $60 \mathrm{~km}$ baseline

\section{May 24 (Low Ionosphere)}

Figure 4 shows the convergence performance for May 24 without stochastic ionospheric modelling. The tightly coupled method converges faster than the other methods, followed by the correction-based approach. The single reference station approach converges very slowly relative to the multiple reference station methods. The individual convergence periods, representative of the 24 periods used to derive Figure 4, are shown in Figure A1 of the Appendix.

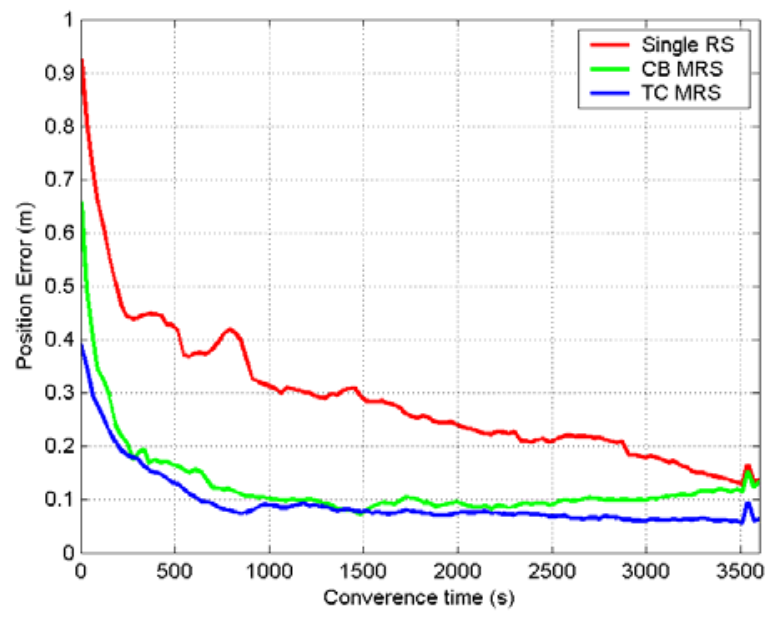

Fig. 4 Convergence of the single reference station, correction-based multiple reference station and tightly coupled approaches for May 24 (low ionosphere) without stochastic ionosphere modelling

Table 1 shows the RMS position errors for the three methods after convergence. The multiple reference station methods perform much better than the single 
reference station approach. This difference is due to network modelling of the ionosphere in the correctionbased approach and ionospheric error averaging in the tightly coupled approach. The results improve drastically when stochastic ionospheric modelling is used.

Table 1 RMS position errors for the single reference station, correctionbased multiple reference station, and tightly coupled approaches, after convergence for May 24 (low ionosphere) without stochastic ionospheric modelling

\begin{tabular}{|l|c|c|c|c|c|}
\hline & $\begin{array}{c}\text { Single } \\
(\mathrm{cm})\end{array}$ & $\begin{array}{c}\text { CB } \\
\text { MRS } \\
(\mathrm{cm})\end{array}$ & $\begin{array}{c}\text { CB MRS } \\
\text { Improvement }\end{array}$ & $\begin{array}{c}\text { TC } \\
\text { MRS } \\
(\mathrm{cm})\end{array}$ & $\begin{array}{c}\text { TC MRS } \\
\text { Improvement }\end{array}$ \\
\hline North & 4.5 & 2.0 & $56 \%$ & 1.8 & $60 \%$ \\
\hline East & 5.2 & 1.2 & $77 \%$ & 1.1 & $79 \%$ \\
\hline Height & 9.3 & 5.3 & $43 \%$ & 5.5 & $41 \%$ \\
\hline 3D & 11.5 & 5.8 & $50 \%$ & 5.9 & $49 \%$ \\
\hline
\end{tabular}

Figure 5 shows the convergence performance for May 24 with stochastic ionospheric modelling. The individual convergence periods, representative of the 24 periods used to derive Figure 5, are shown in Figure A2 of the Appendix. The convergence in general is very good. The tightly coupled method converges faster than the other methods, followed by the correction-base approach. The tightly coupled approach convergences in approximately 1200 seconds, while the correction-based and single reference station approaches converge after 2000 and 3400 seconds, respectively.

The single reference station and tightly coupled methods perform better with ionospheric modelling. The correction-based approach performs very similarly in both cases. This is because the stochastic ionospheric model decreases the degree of freedom of the position estimation adjustment. For example, the single epoch degree of freedom of a single baseline without stochastic ionospheric modelling is $(s-1) * 2-(6+(s-1) * 2)$ where $s$ is the number of satellites. The first term $\{(s-1)$ * 2\} is for the L1 and L2 carrier phase observations together. The number 6 refers to the 3 position and 3 velocity states and $(s-1) * 2$ is for the L1 and L2 ambiguities for each satellite pair. The degree of freedom changes to $(s-1) * 2-(6+(s-1) * 3)$ when the ionosphere is estimated. The degree of freedom is negative which shows that more than one epoch are required to observe all of the estimated parameters. The increase in model noise due to the decrease in the degree of freedom is usually less than the magnitude of the ionosphere biases that are estimated and removed. The network corrections are effective in removing the ionospheric errors from the measurements, which increases the model noise without decreasing the measurement biases.

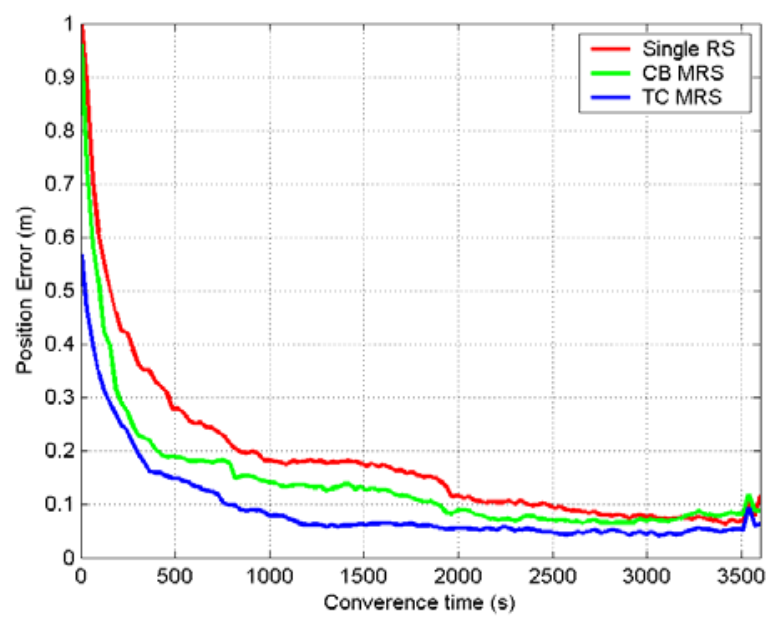

Fig. 5 Convergence of the single reference station, correction-based multiple reference station, and tightly coupled approaches for May 24 (low ionosphere) with stochastic ionosphere modelling.

Table 2 shows the RMS position error for the three methods after convergence. The difference in performance with a low level of ionosphere and stochastic ionospheric modelling is negligible. This is due to the effectiveness of ionospheric modelling.

Table 2 RMS position errors for the single reference station, correctionbased multiple reference station, and tightly coupled approaches after convergence for May 24 (low ionosphere) with stochastic ionosphere modelling.

\begin{tabular}{|l|c|c|c|c|c|}
\hline & $\begin{array}{c}\text { Single } \\
(\mathrm{cm})\end{array}$ & $\begin{array}{c}\text { CB } \\
\text { MRS } \\
(\mathrm{cm})\end{array}$ & $\begin{array}{c}\text { CB MRS } \\
\text { Improvement }\end{array}$ & $\begin{array}{c}\text { TC } \\
\text { MRS } \\
(\mathrm{cm})\end{array}$ & $\begin{array}{c}\text { TC MRS } \\
\text { Improvement }\end{array}$ \\
\hline North & 1.4 & 1.3 & $7 \%$ & 1.2 & $14 \%$ \\
\hline East & 0.8 & 0.8 & $0 \%$ & 0.8 & $0 \%$ \\
\hline Height & 2.7 & 3.0 & $-11 \%$ & 3.1 & $-14 \%$ \\
\hline 3D & 3.2 & 3.3 & $-3 \%$ & 3.4 & $-6 \%$ \\
\hline
\end{tabular}

A comparison of the results from Tables 1 and 2 reveals that the stochastic ionospheric modelling decreases the position RMS by slightly less than half for the multiple reference station methods and more than three times for the single reference station approach. This further shows the effectiveness and importance of ionospheric modelling, even under fairly benign ionospheric conditions.

\section{April 6 (High Ionosphere)}

Figure 6 shows the convergence of the three methods for April 6, which has a high level of ionospheric effect. No ionosphere modelling is applied. The convergence is 
slow in all cases although the multiple reference station methods perform much better than the single reference station approach. The convergence periods used to derive Figure 4, are shown in Figure A3 of the Appendix. Figure A3 shows that the convergence is representative of the ionosphere levels throughout the day.

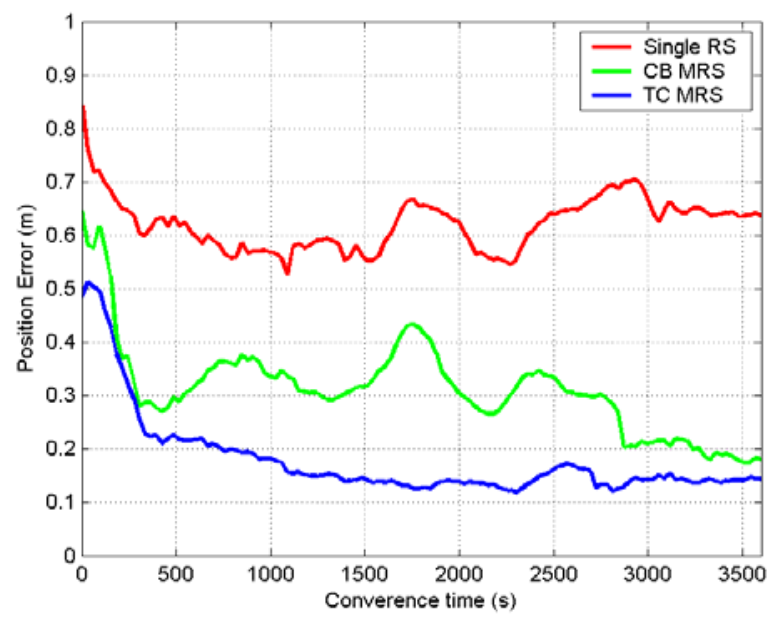

Fig. 6 Convergence of the single reference station, correction-based multiple reference station, and tightly coupled approaches for Apr. 6 (high ionosphere) without stochastic ionosphere modelling.

Figure 7 shows the 3D position errors for the three methods over the entire 24-hour period. The accuracies of the methods are correlated to the magnitude of the differential ionospheric effect shown in Figure 3. The error at the beginning of the data set is up to $2 \mathrm{~m}$ for the single reference station approach. This is due to the large variability of the ionosphere shown in Figure 3. This decreases to less than $10 \mathrm{~cm}$ when the ionospheric effect is lower near the end of the day. The correction-based approach performs best during this test, which shows that the corrections are effective in reducing the effect of the ionosphere. The tightly coupled approach performs better than the single reference station approach but is still affected by the ionosphere.

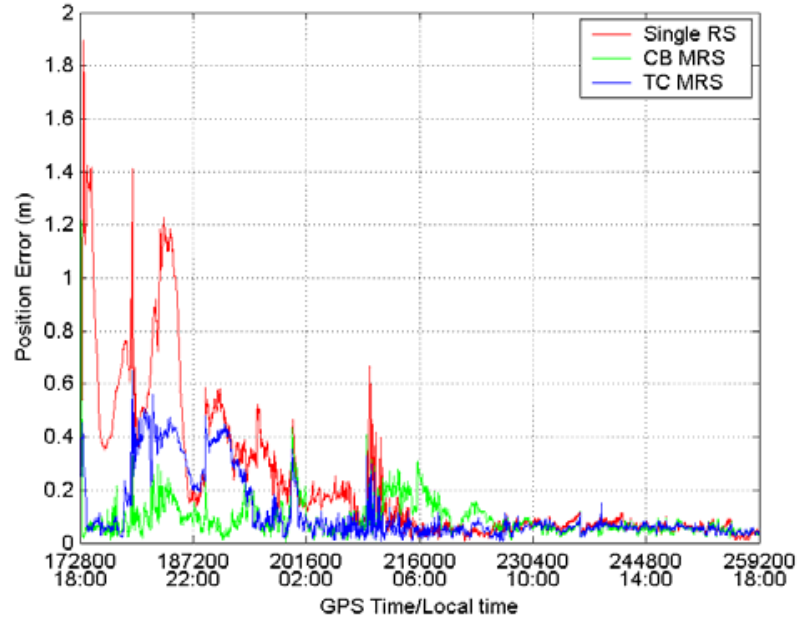

Fig. 7 3D position error over time for the single reference station, correction-based multiple reference station and tightly coupled approaches, for Apr. 6 (high ionosphere) without stochastic ionosphere modelling.

The performance is improved when stochastic ionospheric modelling is applied. Figure 8 shows the convergence of the methods in this case. The individual convergence periods shown in Figure A3 when applying the ionospheric modelling are shown in Figure A4. For this data set the correction-based approach performs slightly better than the tightly coupled approach, which performs significantly better than the single reference station approach. Comparing Figures 6 and 8 shows the improvement due to stochastic ionospheric modelling. Without stochastic ionospheric modelling, none of the methods can achieve an accuracy better than $10 \mathrm{~cm}$ mean position error within the one hour convergence test. When stochastic ionospheric modelling is used, all the methods converge to an accuracy better than $10 \mathrm{~cm}$ in less than 2500 seconds.

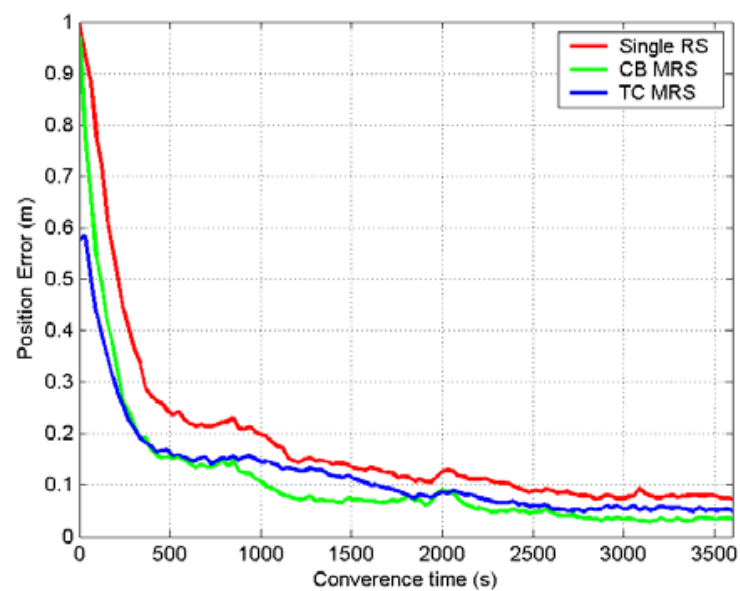

Fig. 8 Convergence of the single reference station, correction-based multiple reference station, and tightly coupled approaches, for Apr. 6 (high ionosphere) with stochastic ionosphere modelling. 
Figure 9 shows the 3D position errors over time with stochastic ionospheric modelling. A comparison with Figure 7 shows a major improvement. Table 3 shows the RMS position errors for the methods after convergence. The position accuracy for all of the methods is exceptional (better than $4 \mathrm{~cm}$ 3D position RMS). Although the multiple reference station methods provide a noticeable reduction of the convergence time there is little if any improvement in the converged position accuracy for this data set.

A comparison of the results of Table 2 and 3 reveals the same level of accuracy for both a low and high level of ionospheric effect when stochastic ionosphere modelling is applied. This somewhat surprising result further confirms the effectiveness of the ionospheric modelling approach used in the software.

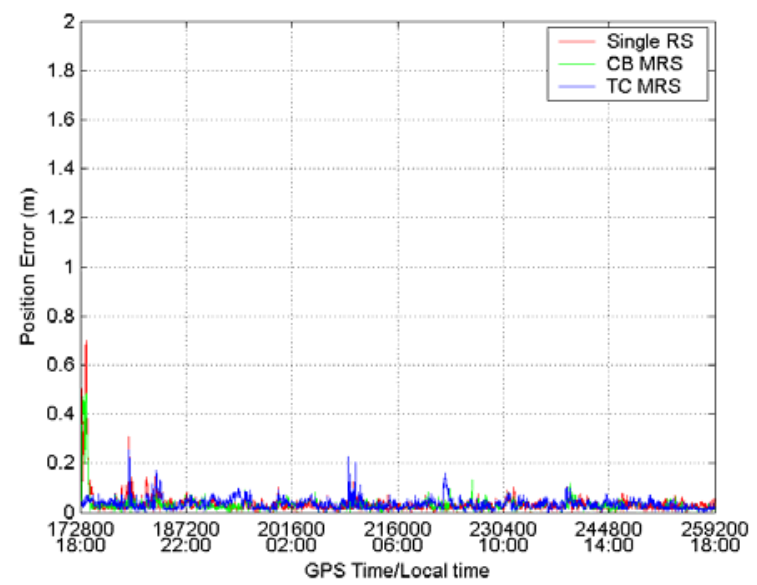

Fig. 9 3D position error over time for the single reference station, correction-based multiple reference station and tightly coupled approaches, for Apr. 6 (high ionosphere) with stochastic ionosphere modelling

\begin{tabular}{|l|c|c|c|c|c|}
\hline & $\begin{array}{c}\text { Single } \\
(\mathrm{cm})\end{array}$ & $\begin{array}{c}\text { CB } \\
\text { MRS } \\
(\mathrm{cm})\end{array}$ & $\begin{array}{c}\text { CB MRS } \\
\text { Improvement }\end{array}$ & $\begin{array}{c}\text { TC } \\
\text { MRS } \\
(\mathrm{cm})\end{array}$ & $\begin{array}{c}\text { TC MRS } \\
\text { Improvement }\end{array}$ \\
\hline North & 1.6 & 1.3 & $19 \%$ & 1.3 & $19 \%$ \\
\hline East & 0.8 & 0.8 & $0 \%$ & 0.9 & $-13 \%$ \\
\hline Height & 3.0 & 2.9 & $3 \%$ & 3.7 & $-23 \%$ \\
\hline 3D & 3.5 & 3.3 & $6 \%$ & 4.0 & $-14 \%$ \\
\hline
\end{tabular}

Table 3 RMS position errors for the single reference station, correctionbased multiple reference station, and tightly coupled approaches after convergence, for Apr 6 (high ionosphere) with stochastic ionosphere modelling

\section{Conclusions}

This paper compares three different carrier phase based DGPS RTK methods, namely the traditional single reference station approach, a collocation-based correction-based multiple reference station approach, and a tightly coupled multiple reference station approach. Two days of data from a medium scale network are used to assess the methods. These days represent a relatively high level and low level of ionosphere activity.

The multiple reference station approaches are effective in reducing convergence time by more than half in some cases. The tightly coupled approach converged slightly faster than the correction-based approach although they both performed well. When using stochastic ionospheric modelling, there was little position accuracy difference between the approaches after convergence. However without stochastic ionospheric modelling, the multiple reference station approaches perform significantly better than the single reference station approach, with a 3D position error reduction of nearly 50 percent.

All the results show a high level of improvement due to stochastic ionospheric modelling in terms of both convergence time and position accuracy during and after convergence. In most of the tests, the use of stochastic ionospheric modelling reduced the position errors by half and sometimes as much at three times. Even with stochastic ionospheric modelling, the multiple reference station approaches provide significant improvements in terms of convergence time relative to the single reference station approach.

\section{References}

Alves P (2004) Development of two novel carrier phase-based methods for multiple Reference station positioning. $\mathrm{PhD}$ Thesis, published as UCGE Report No. 20203, Department of Geomatics Engineering, The University of Calgary.

Alves P, Lachapelle G, and Cannon ME (2004) In-Receiver Multiple Reference Station RTK Solution, Proceedings of the Institute of Navigation Saetllite Division Technical Meeting, GPS 04 (September 2004, Long Beach, USA).

Dao D, Alves P and Lachapelle G (2004) Performance Evaluation of Multiple Reference Station GPS RTK for a Medium Scaled Network. Proceedings of the International Symposium on GPS/GNSS, Sydney, Australia (6-8 December).

Lachapelle G, and Alves P (2002) Multiple Reference Station Approach: Overview and Current Research, Invited Contribution, Expert Forum. Journal of Global Positioning Systems, 1(2), $133-136$.

Lachapelle G, Liu C, and Lu G (1993) Quadruple Single Frequency Receiver System for Ambiguity Resolution on the Fly. Proceedings of the International Technical Meeting of the Satellite Division of the Institute of Navigation, ION GPS 03 (September 1993, Salt Lake City, USA), 1167 - 1172.

Luo N, and Lachapelle G (2003) Relative Positioning of Multiple Moving Platforms using GPS. IEEE 
Transactions on Aerospace and Electronic Systems, 39(3), $936-948$.

Odijk D (1999) Sochastic Modelling of the Ionosphere for Fast GPS Ambiguity Resolution. Proceedings of General Assembly of the International Association of Geodesy (July 1999, Birmingham, England), volume 121, 387 392. International Association of Geodesy, SpringerVerlag.

Odijk D (2000) Weighting Ionospheric Correction to Improve Fast GPS Positioning Over Medium Distances. Proceedings of the National Technical Meeting of the Satellite Division of the Institute of Navigation, ION GPS 2000 (September 2000, Salt Lake, USA), 1113 - 1124.

Pugliano G, Alves P, Cannon ME, and Lachapelle G (2003) Performance Analysis of a Post-Mission Multi-Reference
RTK DGPS Positioning Approach. Proceedings of the International Association of Institutes of Navigation World Congress (October 2003, Berlin, Germany)

Puliano G, Obrizza F, Pingue F, Sepe V, Alves P, and Lachapelle G (2004) Monitoring the Neapolitan Volcanic Area Using an Advanced Multiple Reference Station RTK DGPS Technique, Proceedings of the Institute of Navigation Satellite Division Technical Meeting, GPS 04 (September 2004, Long Beach, USA)

Raquet J (1998) Development of a Method for Kinematic GPS Carrier-Phase Ambiguity Resolution Using Multiple Reference Receivers. PhD Thesis, published as UCGE Report No. 20116, Department of Geomatics Engineering, The University of Calgary.

\section{APPENDIX}

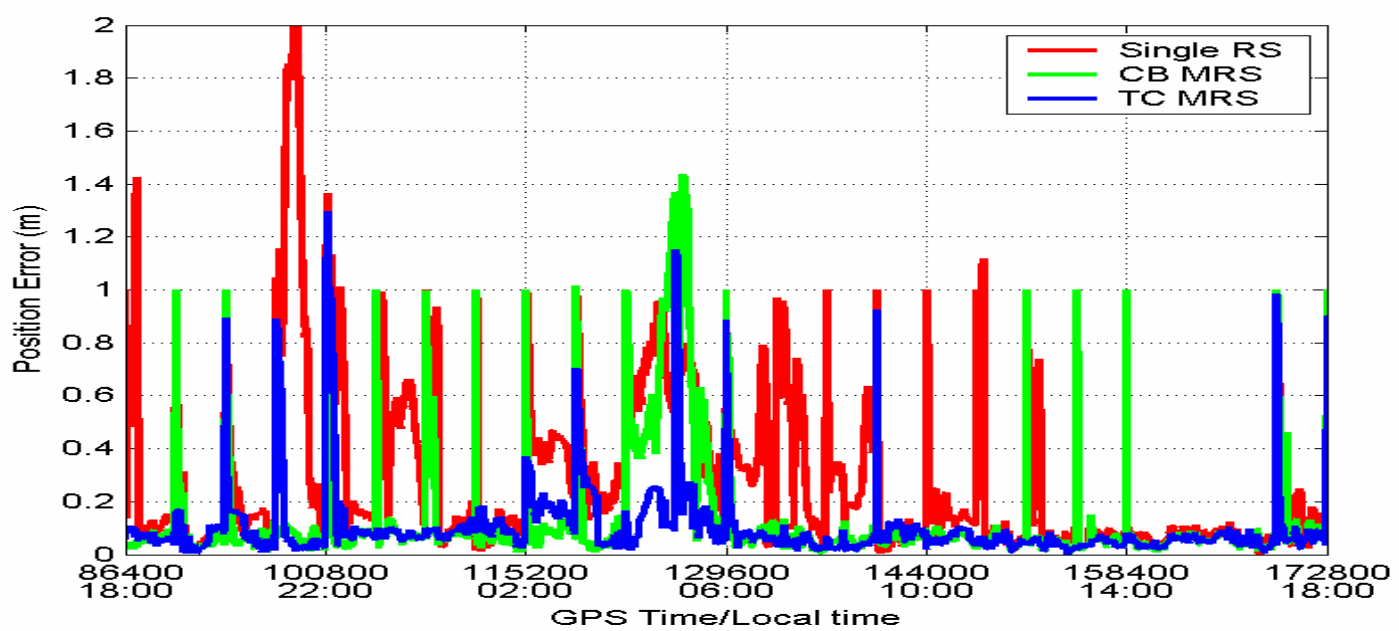

Fig. A1 All convergence periods of the single reference station, correction-based multiple reference station and tightly coupled approaches for May 24 (low ionosphere) without stochastic ionosphere modelling. 


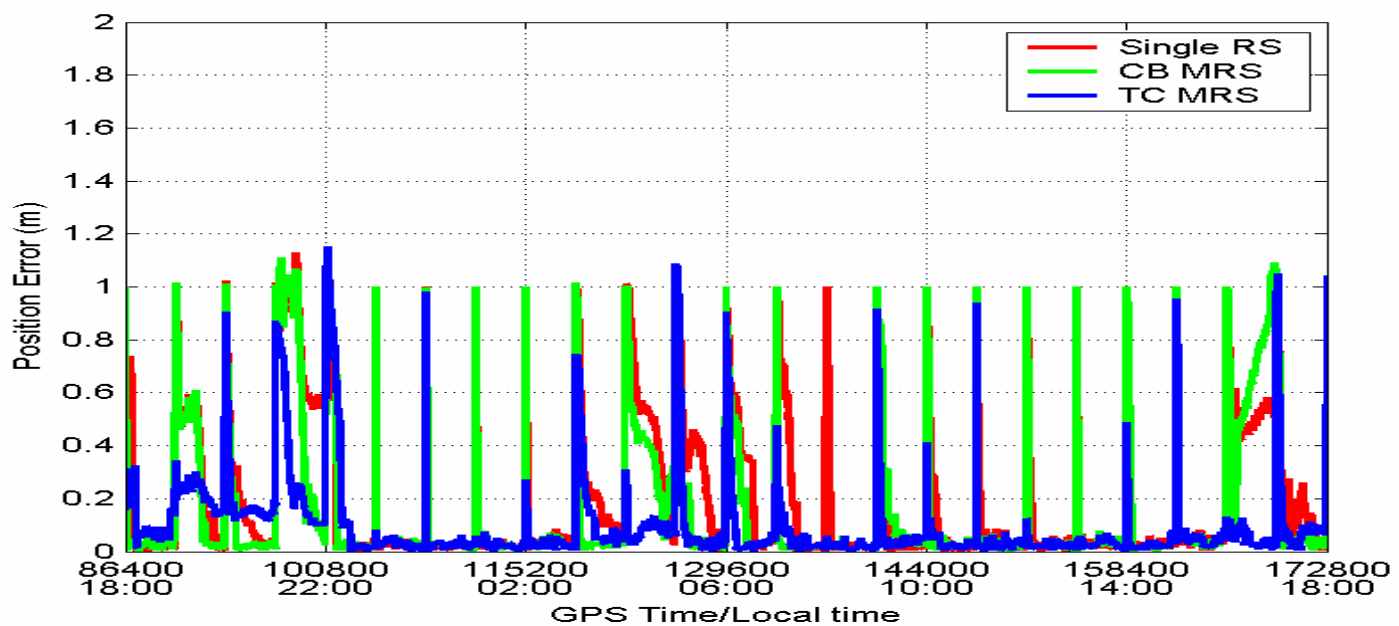

Fig. A2 All convergence periods of the single reference station, correction-based multiple reference station and tightly coupled approaches for May 24 (low ionosphere) with stochastic ionosphere modelling.

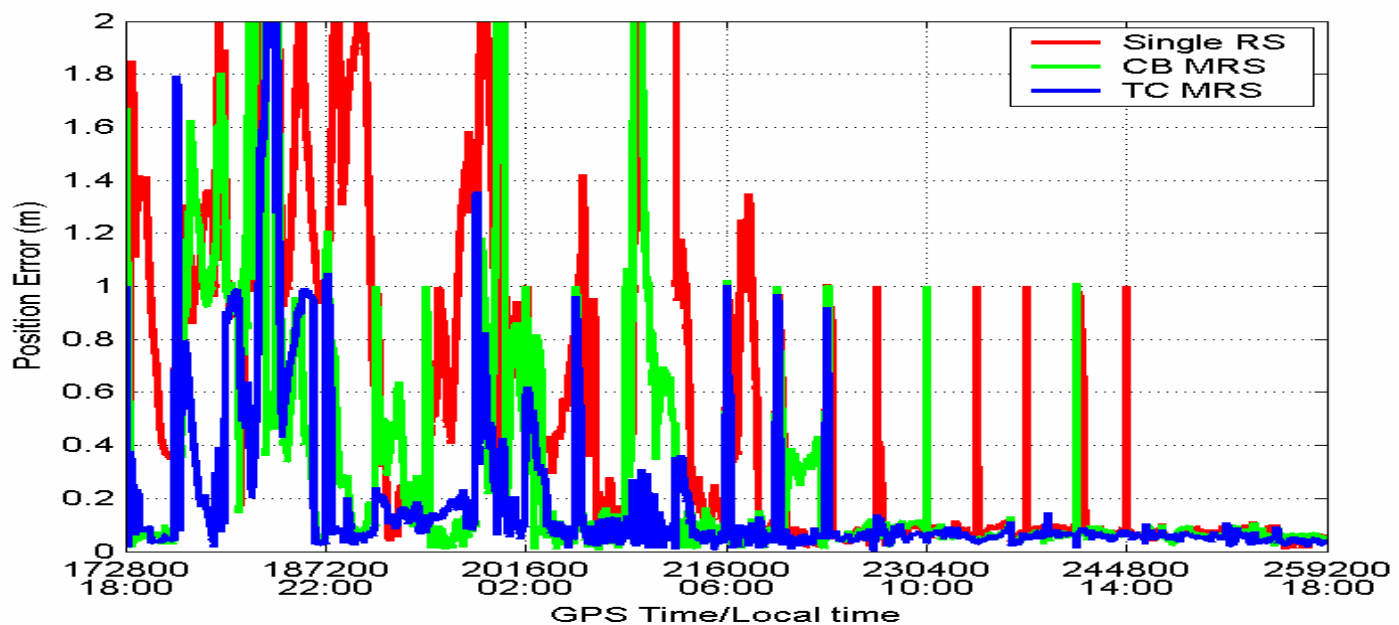

Fig. A3 All convergence periods of the single reference station, correction-based multiple reference station and tightly coupled approaches for April 6 (high ionosphere) without stochastic ionosphere modelling.

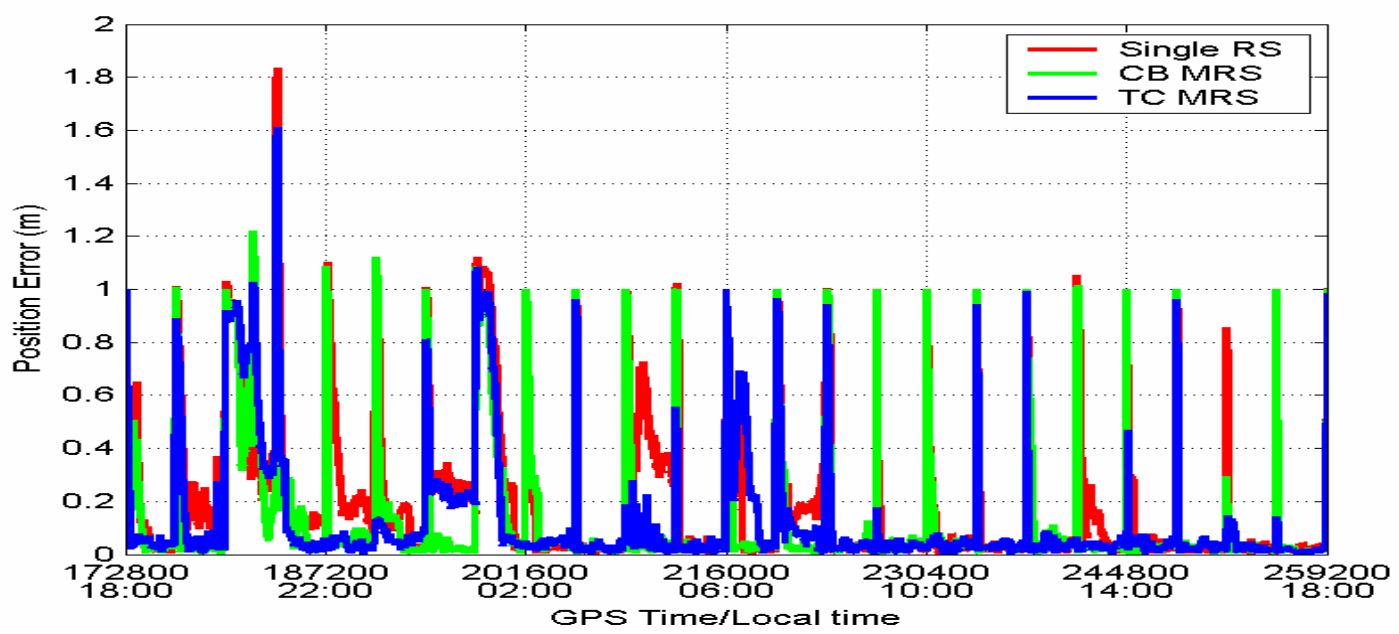

Fig. A4 All convergence periods (same as Figure A2) of the single reference station, correction-based multiple reference station and tightly coupled approaches for April 6 (high ionosphere) with stochastic ionosphere modelling. 